\title{
SOME FUZZY SP-TOPOLOGICAL PROPERTIES
}

\author{
S. E. ABBAS
}

Received 22 January 2003

\begin{abstract}
We introduce $r$-fuzzy strongly preopen and $r$-fuzzy strongly preclosed sets in fuzzy topological space in view of the definition of Šostak (1985). We investigate some properties of them. Moreover, the concepts of fuzzy SP-continuous, fuzzy SP-irresolute continuous, fuzzy SP-irresolute open (closed) mappings, and a fuzzy SP-irresolute homeomorphism are introduced and studied. Some of their characteristic properties are considered.
\end{abstract}

2000 Mathematics Subject Classification: 54A40.

1. Introduction and preliminaries. After the introduction of fuzzy sets by Zadeh [20], Chang [1] was the first to introduce the concept of a fuzzy topology on a set $X$ by axiomatizing a collection $T$ of fuzzy subsets of $X$, where he referred to each member of $T$ as an open set. In his definition of fuzzy topology, fuzziness in the concept of openness of a fuzzy subset was absent. In [2, 4], the authors introduced the concept of gradation of openness (closedness) of fuzzy subsets of $X$ in two different ways and gave definitions of a fuzzy topology on $X$. They referred to the fuzzy topology in the sense of Chang as the topology of fuzzy subsets.

The gradation of openness on $X$, as introduced in [2], is a mapping $\tau: I^{X} \rightarrow I$ satisfying the following conditions:

(O1) $\tau(\underline{0})=\tau(\underline{1})=1$,

(O2) $\tau\left(\mu_{1} \wedge \mu_{2}\right) \geq \tau\left(\mu_{1}\right) \wedge \tau\left(\mu_{2}\right)$, for any $\mu_{1}, \mu_{2} \in I^{X}$,

(O3) $\tau\left(\bigvee_{i \in \Gamma} \mu_{i}\right) \geq \bigwedge_{i \in \Gamma} \tau\left(\mu_{i}\right)$, for any $\left\{\mu_{i}\right\}_{i \in \Gamma} \subset I^{X}$.

The pair $(X, \tau)$ is called a fuzzy topological space (FTS).

Historically, the fundamental idea of a topology itself being fuzzy, that is, a topology being a fuzzy subset of a powerset, first appeared in 1980 in [5], a paper in which a topology was a fuzzy subset of a traditional powerset of crisp subsets and in which the axioms of the preceding paragraph appear for mappings defined on traditional powersets. This was followed in 1985 by the independent and parallel generalizations of Kubiak [11] and Šostak [15], papers in which a topology was a fuzzy subset of a powerset of fuzzy subsets, and in $[11,15]$ appear exactly the axioms of the preceding paragraph. Developments of this idea continued in $[16,17,18]$ for the lattice $L=I$ of membership values, and significant generalizations of the restriction of $L=I$ were given in 1995 in [6] and again in 1997 in [12], where in the latter two, membership lattices were used to describe such a topology, namely, a mapping from $L^{X}$ to $M$, where $L$ and $M$ are appropriate lattices. Finally, we have the foundations of such topologies developed in 1999: axiomatic foundations are laid in [7] for the original idea as given in $[11,15]$ in which the base lattice $L$ is fixed, and categorical foundations are laid in [7] 
for yet another significant generalization, the variable basis case, in which the lattice $L$ varies from space to space.

The board appeal of the notion of a topology as a fuzzy subset of a powerset of fuzzy subsets is being independently considered by authors not aware of the historical development of $[5,6,7,11,12,14,15,16,17,18]$, particularly its roots in $[5,11,15]$, including Chattopadhyay et al. [2], Ramadan [13], and Ying [19].

In this paper, we introduce $r$-fuzzy strongly preopen and $r$-fuzzy strongly preclosed sets in an FTS of Šostak [15] as an extension of those of Krsteska [9, 10]. We investigate some properties of them. The classes of fuzzy strongly precontinuous, fuzzy strongly preirresolute continuous, and fuzzy strongly preirresolute open (closed) mapping are introduced. We show that fuzzy continuity implies fuzzy strong precontinuity and fuzzy strongly preirresolute continuity implies fuzzy strong precontinuity, but the inverse of them is not true. Also, we obtain some properties of fuzzy strongly preirresolute continuous mappings.

Throughout this paper, let $X$ be a nonempty set, $I=[0,1]$, and $I_{\circ}=(0,1]$. For $\alpha \in I$, $\underline{\alpha}(x)=\alpha$ for all $x \in X$.

THEOREM 1.1 [3]. Let $(X, \tau)$ be an FTS. Then for each $r \in I_{\circ}, \lambda \in I^{X}$, we define an $r$-fuzzy closure operator $C_{\tau}: I^{X} \times I_{\circ} \rightarrow I^{X}$ as follows:

$$
C_{\tau}(\lambda, r)=\bigwedge\left\{\mu \in I^{X}: \lambda \leq \mu, \tau(\underline{1}-\mu) \geq r\right\} .
$$

For $\lambda, \mu \in I^{X}$ and $r, s \in I_{\circ}$, the operator $C_{T}$ satisfies the following conditions:

(1) $C_{T}(\underline{0}, r)=\underline{0}$,

(2) $\lambda \leq C_{T}(\lambda, r)$,

(3) $C_{T}(\lambda, r) \vee C_{T}(\mu, r)=C_{T}(\lambda \vee \mu, r)$,

(4) $C_{T}(\lambda, r) \leq C_{T}(\lambda, s)$ if $r \leq s$,

(5) $C_{T}\left(C_{T}(\lambda, r), r\right)=C_{\tau}(\lambda, r)$.

THEOREM 1.2 [8]. Let $(X, \tau)$ be an FTS. Then, for each $r \in I_{\circ}, \lambda \in I^{X}$, we define an $r$-fuzzy interior operator $I_{\tau}: I^{X} \times I_{\circ} \rightarrow I^{X}$ as follows:

$$
I_{\tau}(\lambda, r)=\bigvee\left\{\mu \in I^{X}: \lambda \geq \mu, \tau(\mu) \geq r\right\}
$$

For $\lambda, \mu \in I^{X}$ and $r, s \in I_{\circ}$, the operator $I_{\tau}$ satisfies the following conditions:

(1) $I_{\tau}(\underline{1}-\lambda, r)=\underline{1}-C_{\tau}(\lambda, r)$,

(2) $I_{\tau}(\underline{1}, r)=\underline{1}$

(3) $\lambda \geq I_{\tau}(\lambda, r)$,

(4) $I_{\tau}(\lambda, r) \wedge I_{\tau}(\mu, r)=I_{\tau}(\lambda \wedge \mu, r)$,

(5) $I_{\tau}(\lambda, r) \geq I_{\tau}(\lambda, s)$ if $r \leq s$,

(6) $I_{\tau}\left(I_{\tau}(\lambda, r), r\right)=I_{\tau}(\lambda, r)$.

DEFINITION 1.3 [8]. Let $(X, \tau)$ be an FTS, for $\lambda \in I^{X}$ and $r \in I_{\circ}$.

(1) $\lambda$ is called $r$-fuzzy semiopen ( $r$-fso, for short) if and only if $\lambda \leq C_{\tau}\left(I_{\tau}(\lambda, r), r\right)$, and $\lambda$ is called $r$-fuzzy semiclosed ( $r$-fsc, for short) if and only if $\underline{1}-\lambda$ is $r$-fso. 
(2) $\lambda$ is called $r$-fuzzy strongly semiopen ( $r$-fsso, for short) if and only if $\lambda \leq$ $I_{T}\left(C_{T}\left(I_{\tau}(\lambda, r), r\right), r\right)$, and $\lambda$ is called $r$-fuzzy strongly semiclosed $(r$-fssc, for short) if and only if $\underline{1}-\lambda$ is $r$-fsso.

(3) $\lambda$ is called $r$-fuzzy preopen ( $r$-fpo, for short) if and only if $\lambda \leq I_{\tau}\left(C_{\tau}(\lambda, r), r\right)$, and $\lambda$ is called $r$-fuzzy preclosed ( $r$-fpc, for short) if and only if $\underline{1}-\lambda$ is $r$-fpo.

(4) The $r$-fuzzy preinterior of $\lambda$, denoted by $P I_{\tau}(\lambda, r)$, is defined by $P I_{\tau}(\lambda, r)=$ $\vee\left\{v \in I^{X}: v \leq \lambda, v\right.$ is $r$-fpo $\}$.

(5) The $r$-fuzzy preclosure of $\lambda$, denoted by $P C_{\tau}(\lambda, r)$, is defined by $P C_{\tau}(\lambda, r)=$ $\bigwedge\left\{v \in I^{X}: v \geq \lambda, v\right.$ is $r$-fpc $\}$.

LEMMA 1.4. Let $(X, \tau)$ be an FTS for $\lambda \in I^{X}$ and $r \in I_{\circ}$. Then,

(1) $\lambda \vee C_{T}\left(I_{\tau}(\lambda, r), r\right) \leq P C_{T}(\lambda, r)$,

(2) $P I_{T}(\lambda, r) \leq \lambda \wedge I_{\tau}\left(C_{T}(\lambda, r), r\right)$,

(3) $I_{T}\left(P C_{T}(\lambda, r), r\right) \leq I_{\tau}\left(C_{T}(\lambda, r), r\right)$,

(4) $I_{\tau}\left(C_{T}\left(I_{\tau}(\lambda, r), r\right), r\right) \leq I_{\tau}\left(P C_{T}(\lambda, r), r\right)$,

(5) $P C_{T}(\underline{1}-\lambda, r)=\underline{1}-P I_{\tau}(\lambda, r)$ and $P I_{\tau}(\underline{1}-\lambda, r)=\underline{1}-P C_{T}(\lambda, r)$.

Proof. (1) Since $P C_{\tau}(\lambda, r)$ is $r$-fpc, we have

$$
C_{\tau}\left(I_{\tau}(\lambda, r), r\right) \leq C_{\tau}\left(I_{\tau}\left(P C_{\tau}(\lambda, r), r\right), r\right) \leq P C_{\tau}(\lambda, r)
$$

Thus, $\lambda \vee C_{\tau}\left(I_{\tau}(\lambda, r), r\right) \leq P C_{T}(\lambda, r)$.

(2) It can be shown as (1).

(3) It follows from the relation $P C_{\tau}(\lambda, r) \leq C_{T}(\lambda, r)$.

(4) From (1) we have

$$
I_{\tau}\left(P C_{\tau}(\lambda, r), r\right) \geq I_{\tau}\left(\lambda \vee C_{\tau}\left(I_{\tau}(\lambda, r), r\right), r\right) \geq I_{\tau}\left(C_{\tau}\left(I_{\tau}(\lambda, r), r\right), r\right) .
$$

(5) It is straightforward.

Definition $1.5[8,15]$. Let $(X, \tau)$ and $(Y, \eta)$ be FTS's. Let $f: X \rightarrow Y$ be a mapping.

(1) $f$ is called fuzzy continuous if and only if $\eta(\mu) \leq \tau\left(f^{-1}(\mu)\right)$ for each $\mu \in I^{Y}$.

(2) $f$ is called fuzzy open if and only if $\tau(\lambda) \leq \eta(f(\lambda))$ for each $\lambda \in I^{X}$.

(3) $f$ is called fuzzy closed if and only if $\tau(\underline{1}-\lambda) \leq \eta(\underline{1}-f(\lambda))$ for each $\lambda \in I^{X}$.

(4) $f$ is called fuzzy strongly semicontinuous (resp., fuzzy semicontinuous and fuzzy precontinuous) if and only if $f^{-1}(\mu)$ is an $r$-fsso (resp., $r$-fso and $r$-fpo) set of $X$ for each $\mu \in I^{Y}, r \in I$ 。 with $\eta(\mu) \geq r$.

\section{2. $r$-fuzzy strongly preopen and $r$-fuzzy strongly preclosed sets}

DEFINITION 2.1. Let $(X, \tau)$ be an FTS for $\lambda \in I^{X}$ and $r \in I_{\circ}$.

(1) $\lambda$ is called $r$-fuzzy strongly preopen ( $r$-fspo, for short) if and only if $\lambda \leq I_{\tau}\left(P C_{\tau}(\lambda\right.$, $r), r)$.

(2) $\lambda$ is called $r$-fuzzy strongly preclosed ( $r$-fspc, for short) if and only if $\underline{1}-\lambda$ is $r$-fspo. Equivalently, $C_{T}\left(P I_{T}(\lambda, r), r\right) \leq \lambda$.

(3) The $r$-fuzzy strongly preinterior of $\lambda$, denoted by $\operatorname{SPI}_{\tau}(\lambda, r)$, is defined by $\operatorname{SPI}_{\tau}(\lambda, r)=\bigvee\left\{v \in I^{X}: v \leq \lambda, v\right.$ is $r$-fspo $\}$. 
(4) The $r$-fuzzy strongly preclosure of $\lambda$, denoted by $\operatorname{SPC}_{T}(\lambda, r)$, is defined by $\operatorname{SPC}_{T}(\lambda, r)=\bigwedge\left\{v \in I^{X}: v \geq \lambda, v\right.$ is $r$-fspc $\}$.

THEOREM 2.2. Let $(X, \tau)$ be an FTS. For $\lambda, \mu \in I^{X}$ and $r \in I_{\circ}$,

(1) if $\tau(\lambda) \geq r$, then $\lambda$ is $r$-fspo,

(2) if $\lambda$ is $r$-fsso, then $\lambda$ is $r$-fspo,

(3) if $\lambda$ is $r$-fspo, then $\lambda$ is $r$-fpo,

(4) $\lambda$ is $r$-fspo if and only if there exists $\mu \in I^{X}$ such that $\mu \leq \lambda \leq I_{\tau}\left(P C_{\tau}(\mu, r), r\right)$,

(5) $\lambda$ is $r$-fspc if and only if there exists $\mu \in I^{X}$ such that $C_{\tau}\left(P I_{\tau}(\mu, r), r\right) \leq \lambda \leq \mu$.

Proof. (1), (2), and (3) are easily proved from Lemma 1.4.

(4) Let $\lambda \in I^{X}$. If $\mu \in I^{X}$ such that $\mu \leq \lambda \leq I_{T}\left(P C_{T}(\mu, r), r\right)$ exists, then $\lambda \leq$ $I_{\tau}\left(P C_{T}(\mu, r), r\right) \leq I_{\tau}\left(P C_{\tau}(\lambda, r), r\right)$. Thus $\lambda$ is $r$-fspo. Conversely, if $\lambda$ is $r$-fspo, then the result follows for $\lambda=\mu$.

(5) Similar to the proof of (4).

THEOREM 2.3. Let $(X, \tau)$ be an FTS. For $r \in I_{\circ}$,

(1) any union of $r$-fspo sets is $r$-fspo,

(2) any intersection of $r$-fspc sets is $r$-fspc.

Proof. (1) Let $\left\{\lambda_{\alpha}: \alpha \in \Gamma\right\}$ be a family of $r$-fspo sets. For each $\alpha \in \Gamma, \lambda_{\alpha} \leq I_{\tau}\left(P C_{\tau}\left(\lambda_{\alpha}\right.\right.$, $r), r)$. Hence, we have

$$
\bigvee_{\alpha \in \Gamma} \lambda_{\alpha} \leq \bigvee_{\alpha \in \Gamma}\left(I_{\tau}\left(P C_{\tau}\left(\lambda_{\alpha}, r\right), r\right)\right) \leq I_{\tau}\left(P C_{\tau}\left(\bigvee_{\alpha \in \Gamma} \lambda_{\alpha}, r\right), r\right)
$$

So, $V_{\alpha \in \Gamma} \lambda_{\alpha}$ is $r$-fspo.

(2) Similar to the proof of (1).

REMARK 2.4. The intersection of two $r$-fspo sets need not be $r$-fspo. The union of two $r$-fspc sets need not be $r$-fspc. We will show it from Example 2.6(3).

THEOREM 2.5. Let $(X, \tau)$ be an FTS. For $\lambda, \mu \in I^{X}$ and $r \in I_{\circ}$, the following statements hold:

(1) $C_{\tau}(\lambda, r)$ is $r$-fspc,

(2) $\lambda$ is $r$-fspo if and only if $\lambda=\operatorname{SPI}_{\tau}(\lambda, r)$,

(3) $\lambda$ is $r$-fspc if and only if $\lambda=\operatorname{SPC}_{T}(\lambda, r)$,

(4) $I_{\tau}(\lambda, r) \leq \operatorname{SPI}_{\tau}(\lambda, r) \leq P I_{\tau}(\lambda, r) \leq \lambda \leq P C_{\tau}(\lambda, r) \leq \operatorname{SPC}_{\tau}(\lambda, r) \leq C_{\tau}(\lambda, r)$,

(5) $\mathrm{SPI}_{\tau}(\underline{1}-\lambda, r)=\underline{1}-\mathrm{SPC}_{T}(\lambda, r)$ and $\mathrm{SPC}_{T}(\underline{1}-\lambda, r)=\underline{1}-\mathrm{SPI}_{T}(\lambda, r)$,

(6) $C_{T}\left(\operatorname{SPC}_{T}(\lambda, r), r\right)=\operatorname{SPC}_{T}\left(C_{T}(\lambda, r), r\right)=C_{T}(\lambda, r)$.

Proof. (1), (2), (3), (4) follow from the definitions.

(5) For all $\lambda \in I^{X}, r \in I_{\circ}$, we have the following:

$$
\begin{aligned}
\underline{1}-\operatorname{SPI}_{\tau}(\lambda, r) & =\underline{1}-\bigvee\{v: v \leq \lambda, v \text { is } r \text {-fspo }\} \\
& =\bigwedge\{\underline{1}-v: \underline{1}-\lambda \leq \underline{1}-v, \underline{1}-v \text { is } r \text {-fspc }\} \\
& =\operatorname{SPC}_{\tau}(\underline{1}-\lambda, r) .
\end{aligned}
$$


(6) From Theorem 2.5(1), (3), $\mathrm{SPC}_{\tau}\left(C_{\tau}(\lambda, r), r\right)=C_{\tau}(\lambda, r)$. We only show that

$$
C_{\tau}\left(\mathrm{SPC}_{\tau}(\lambda, r), r\right)=C_{\tau}(\lambda, r) .
$$

Since $\lambda \leq \operatorname{SPC}_{\tau}(\lambda, r)$,

$$
C_{T}\left(\mathrm{SPC}_{\tau}(\lambda, r), r\right) \geq C_{\tau}(\lambda, r)
$$

Suppose that

$$
C_{T}\left(\operatorname{SPC}_{\tau}(\lambda, r), r\right) \npreceq C_{\tau}(\lambda, r) .
$$

There exist $x \in X$ and $r \in I$ 。 such that

$$
C_{T}\left(\operatorname{SPC}_{\tau}(\lambda, r), r\right)(x)>C_{\tau}(\lambda, r)(x) .
$$

By the definition of $C_{\tau}$, there exists $\rho \in I^{X}$ with $\lambda \leq \rho$ and $\tau(\underline{1}-\rho) \geq r$ such that

$$
C_{\tau}\left(\mathrm{SPC}_{\tau}(\lambda, r), r\right)(x)>\rho(x) \geq C_{\tau}(\lambda, r)(x) .
$$

On the other hand, since $\rho=C_{\tau}(\rho, r), \lambda \leq \rho$ implies

$$
\operatorname{SPC}_{\tau}(\lambda, r) \leq \operatorname{SPC}_{\tau}(\rho, r)=\operatorname{SPC}_{\tau}\left(C_{\tau}(\rho, r), r\right)=C_{\tau}(\rho, r)=\rho .
$$

Thus,

$$
C_{T}\left(\mathrm{SPC}_{T}(\lambda, r), r\right) \leq \rho .
$$

It is a contradiction. Hence, $C_{T}\left(\operatorname{SPC}_{\tau}(\lambda, r), r\right) \leq C_{T}(\lambda, r)$.

EXAMPLE 2.6. Let $X=\{a, b, c\}$. Define the fuzzy sets $\mu, \rho, v \in I^{X}$ as follows:

$$
\begin{aligned}
& \mu(a)=0.3, \quad \mu(b)=0.2, \quad \mu(c)=0.7, \\
& \rho(a)=0.8, \quad \rho(b)=0.8, \quad \rho(c)=0.4 \text {, } \\
& v(a)=0.8, \quad v(b)=0.7, \quad v(c)=0.6 \text {. }
\end{aligned}
$$

Define the fuzzy topology $\tau: I^{X} \rightarrow I$ as follows:

$$
\tau(\lambda)= \begin{cases}1, & \text { if } \lambda=\underline{0}, \underline{1}, \\ \frac{1}{2}, & \text { if } \lambda=\mu, \\ \frac{2}{3}, & \text { if } \lambda=\rho, \\ \frac{2}{3}, & \text { if } \lambda=\mu \wedge \rho, \\ \frac{1}{2}, & \text { if } \lambda=\mu \vee \rho, \\ 0, & \text { otherwise. }\end{cases}
$$


(1) By easy verification, it can be seen that $\underline{1}=P C_{\tau}(\nu, 1 / 2)>v \vee C_{\tau}\left(I_{\tau}(\nu, 1 / 2), 1 / 2\right)=$ $\{0.8,0.8,0.6\}$ and $P I_{T}(\underline{1}-v, r)<\underline{1}-v \wedge I_{\tau}\left(C_{T}(\underline{1}-v, 1 / 2), 1 / 2\right)$.

(2) $v$ is $(1 / 2)$-fspo set, but $v$ is not a $(1 / 2)$-fsso set.

(3) Let $\eta \in I^{X}$ as $\eta(a)=0.4, \eta(b)=0.2, \eta(c)=0.8$, then $\eta$ is (1/2)-fspo set, but $\rho \wedge \eta$ is not a $(1 / 2)$-fspo set of $(X, \tau)$ because

$$
\begin{gathered}
\eta \leq I_{\tau}\left(P C_{\tau}\left(\eta, \frac{1}{2}\right), \frac{1}{2}\right)=I_{\tau}\left(\underline{1}, \frac{1}{2}\right)=\underline{1}, \\
\rho \wedge \eta \nless I_{\tau}\left(P C_{\tau}\left(\rho \wedge \eta, \frac{1}{2}\right), \frac{1}{2}\right)=I_{\tau}\left(\underline{1}-\mu \wedge \rho, \frac{1}{2}\right)=\mu \wedge \rho .
\end{gathered}
$$

(4) If we define $\sigma: I^{X} \rightarrow I$ as

$$
\sigma(\lambda)= \begin{cases}1, & \text { if } \lambda=\underline{0}, \underline{1} \\ \frac{2}{3}, & \text { if } \lambda=\rho \\ 0, & \text { otherwise }\end{cases}
$$

then $\mu$ is a (2/3)-fpo set, which is not (2/3)-fspo because

$$
\begin{aligned}
& \mu \leq I_{\sigma}\left(C_{\sigma}\left(\mu, \frac{2}{3}\right), \frac{2}{3}\right)=I_{\sigma}\left(\underline{1}-\frac{2}{3}\right)=\underline{1}, \\
& \mu \nless I_{\sigma}\left(P C_{\sigma}\left(\mu, \frac{2}{3}\right), \frac{2}{3}\right)=I_{\sigma}\left(\mu, \frac{2}{3}\right)=\underline{0} .
\end{aligned}
$$

REMARK 2.7. From the above example it is not difficult to conclude that an $r$-fpo set may not be an $r$-fspo set, and an $r$-fspo set may not be an $r$-fsso set. Also, the classes of $r$-fspo sets and $r$-fso sets are independent.

\section{Fuzzy SP irresolute continuous mappings}

Definition 3.1. Let $(X, \tau)$ and $(Y, \eta)$ be FTS's. Let $f: X \rightarrow Y$ be a mapping.

(1) $f$ is called fuzzy strongly precontinuous (fuzzy SP continuous, for short) if and only if $f^{-1}(\mu)$ is an $r$-fspo set of $X$ for each $\mu \in I^{Y}, r \in I$ 。 with $\eta(\mu) \geq r$.

(2) $f$ is called fuzzy strongly preirresolute (fuzzy SP irresolute, for short) continuous if and only if $f^{-1}(\mu)$ is an $r$-fspo set of $X$ for each $r$-fspo $\mu \in I^{Y}$.

(3) $f$ is called fuzzy SP irresolute open (resp., fuzzy SP open) if and only if $f(\lambda)$ is an $r$-fspo set of $Y$ for each $r$-fspo $\lambda \in I^{Y}$ (resp., $\tau(\lambda) \geq r$ ).

(4) $f$ is called fuzzy SP irresolute closed (resp., fuzzy SP closed) if and only if $f(\lambda)$ is an $r$-fspc set of $Y$ for each $r$-fspc $\lambda \in I^{Y}$ (resp., $\tau(\underline{1}-\lambda) \geq r$ ).

(5) $f$ is called a fuzzy SP irresolute homeomorphism if and only if $f$ is bijective and both of $f$ and $f^{-1}$ are fuzzy SP irresolute continuous.

REMARK 3.2. (1) Every fuzzy continuous (resp., fuzzy open and fuzzy closed) mapping is fuzzy SP continuous (resp., fuzzy SP open and fuzzy SP closed).

(2) Every fuzzy SP irresolute continuous mapping is fuzzy SP continuous from Theorem 2.2(1). Also, every fuzzy strongly semicontinuous mapping is fuzzy SP continuous. However, the converse of (1) and (2) may be false, see Examples 3.3 and 3.4. 
(3) Fuzzy SP irresolute continuous and fuzzy continuous mappings are independent notions.

EXAMPLE 3.3. We consider Example 2.6. If we put

$$
\eta(\lambda)= \begin{cases}1, & \text { if } \lambda=\underline{0}, \underline{1} \\ \frac{1}{3}, & \text { if } \lambda=\mu \\ 0, & \text { otherwise }\end{cases}
$$

then $\operatorname{id}_{X}:(X, \tau) \rightarrow(X, \eta)$ is fuzzy precontinuous but $\operatorname{id}_{X}$ is not fuzzy SP continuous. Also, if we put

$$
\sigma(\lambda)= \begin{cases}1, & \text { if } \lambda=\underline{0}, \underline{1} \\ \frac{1}{2}, & \text { if } \lambda=v \\ 0, & \text { otherwise }\end{cases}
$$

we obtain $P C_{\sigma}: I^{X} \times I_{\circ} \rightarrow I^{X}$ as follows:

$$
P C_{\sigma}(\lambda, r)= \begin{cases}\underline{1}, & \text { if } \lambda \geq v, \quad 0<r \leq \frac{1}{3}, \\ \lambda, & \text { otherwise. }\end{cases}
$$

Moreover, for each $\lambda \geq v$ and $0<r \leq 1 / 3, \lambda$ is $r$-fspo in $(X, \sigma)$ and $(X, \eta)$. Thus, the identity mapping $\operatorname{id}_{X}:(X, \tau) \rightarrow(X, \sigma)$ is fuzzy SP precontinuous and fuzzy SP irresolute continuous, but $\operatorname{id}_{X}$ is neither fuzzy continuous nor fuzzy strong semicontinuous.

EXAMPLE 3.4. Let $X=\{a, b, c\}$. Define the fuzzy sets $\mu_{1}, \mu_{2}, \mu_{3}, \mu_{4} \in I^{X}$ as follows:

$$
\begin{array}{lll}
\mu_{1}(a)=0.5, & \mu_{1}(b)=0.3, & \mu_{1}(c)=0.6, \\
\mu_{2}(a)=0.3, & \mu_{2}(b)=0.4, & \mu_{2}(c)=0.3, \\
\mu_{3}(a)=0.5, & \mu_{3}(b)=0.4, & \mu_{3}(c)=0.6, \\
\mu_{4}(a)=0.5, & \mu_{4}(b)=0.5, & \mu_{4}(c)=0.6 .
\end{array}
$$

Define fuzzy topologies $\tau, \eta: I^{X} \rightarrow I$ as follows:

$$
\tau(\lambda)=\left\{\begin{array}{ll}
1, & \text { if } \lambda=\underline{0}, \underline{1}, \\
\frac{1}{2}, & \text { if } \lambda=\mu_{1}, \\
\frac{1}{2}, & \text { if } \lambda=\mu_{2}, \\
\frac{2}{3}, & \text { if } \lambda=\mu_{1} \wedge \mu_{2}, \\
\frac{2}{3}, & \text { if } \lambda=\mu_{1} \vee \mu_{2}, \\
0, & \text { otherwise, }
\end{array} \quad \eta(\lambda)= \begin{cases}1, & \text { if } \lambda=\underline{0}, \underline{1}, \\
\frac{1}{3}, & \text { if } \lambda=\mu_{3}, \\
0, & \text { otherwise. }\end{cases}\right.
$$


Then $\operatorname{id}_{X}:(X, \tau) \rightarrow(X, \eta)$ is fuzzy SP continuous but not fuzzy SP irresolute continuous. Furthermore, $\mathrm{id}_{X}$ is a fuzzy continuous mapping which is not fuzzy SP irresolute continuous because

$$
\begin{gathered}
\mu_{4} \leq I_{\eta}\left(P C_{\eta}\left(\mu_{4}, \frac{1}{3}\right), \frac{1}{3}\right)=\underline{1}, \\
\mu_{4} \nless I_{\tau}\left(P C_{\tau}\left(\mu_{4}, \frac{1}{3}\right), \frac{1}{3}\right)=I_{\tau}\left(\underline{1}-\mu_{2}, \frac{1}{3}\right)=\mu_{3} .
\end{gathered}
$$

THEOREM 3.5. Let $\left(X, \tau_{1}\right)$ and $\left(Y, \tau_{2}\right)$ be FTS's. Let $f: X \rightarrow Y$ be a mapping. The following statements are equivalent:

(1) $f$ is fuzzy SP irresolute continuous,

(2) for each $r$-fspc $\mu \in I^{Y}, f^{-1}(\mu)$ is $r$ - $f s p c$,

(3) $f\left(\operatorname{SPC}_{\tau_{1}}(\lambda, r)\right) \leq \operatorname{SPC}_{T_{2}}(f(\lambda), r)$, for each $\lambda \in I^{X}$ and $r \in I_{\circ}$,

(4) $\operatorname{SPC}_{T_{1}}\left(f^{-1}(\mu), r\right) \leq f^{-1}\left(\operatorname{SPC}_{T_{2}}(\mu, r)\right)$, for each $\mu \in I^{Y}$ and $r \in I_{\circ}$,

(5) $f^{-1}\left(\operatorname{SPI}_{\tau_{2}}(\mu, r)\right) \leq \operatorname{SPI}_{\tau_{1}}\left(f^{-1}(\mu), r\right)$, for each $\mu \in I^{Y}$ and $r \in I_{\circ}$,

(6) $C_{T_{1}}\left(P I_{\tau_{1}}\left(f^{-1}(\mu), r\right), r\right) \leq f^{-1}\left(\operatorname{SPC}_{T_{2}}(\mu, r)\right)$, for each $\mu \in I^{Y}$ and $r \in I_{\circ}$,

(7) $f^{-1}\left(\mathrm{SPI}_{\tau_{2}}(\mu, r)\right) \leq I_{\tau_{1}}\left(P C_{\tau_{1}}\left(f^{-1}(\mu), r\right), r\right)$, for each $\mu \in I^{Y}$ and $r \in I_{\circ}$,

(8) $f\left(C_{T_{1}}\left(P I_{T_{1}}(\lambda, r), r\right)\right) \leq \operatorname{SPC}_{T_{2}}(f(\lambda), r)$, for each $\lambda \in I^{X}$ and $r \in I_{\circ}$.

Proof. $(1) \Leftrightarrow(2)$. It is easily proved from Definition 2.1 , and $f^{-1}(\underline{1}-\mu)=\underline{1}-f^{-1}(\mu)$. (2) $\Rightarrow(3)$. Suppose there exist $\lambda \in I^{X}$ and $r \in I$ 。 such that

$$
f\left(\mathrm{SPC}_{\tau_{1}}(\lambda, r)\right) \npreceq \mathrm{SPC}_{\mathrm{T}_{2}}(f(\lambda), r) .
$$

There exist $y \in Y$ and $t \in I_{\circ}$ such that

$$
f\left(\operatorname{SPC}_{\tau_{1}}(\lambda, r)\right)(y)>t>\operatorname{SPC}_{\tau_{2}}(f(\lambda), r)(y)
$$

If $f^{-1}(\{y\})=\phi$, it is a contradiction because $f\left(\operatorname{SPC}_{\tau_{1}}(\lambda, r)\right)(y)=0$.

If $f^{-1}(\{y\}) \neq \phi$, there exists $x \in f^{-1}(\{y\})$ such that

$$
f\left(\operatorname{SPC}_{\tau_{1}}(\lambda, r)\right)(y) \geq \operatorname{SPC}_{\tau_{1}}(\lambda, r)(x)>t>\operatorname{SPC}_{\tau_{2}}(f(\lambda), r)(f(x)) .
$$

Since $\operatorname{SPC}_{\tau_{2}}(f(\lambda), r)(f(x))<t$, there exists $r$-fspc $\mu \in I^{Y}$ with $f(\lambda) \leq \mu$ such that

$$
\operatorname{SPC}_{T_{2}}(f(\lambda), r)(f(x)) \leq \mu(f(x))<t .
$$

Moreover, $f(\lambda) \leq \mu$ implies $\lambda \leq f^{-1}(\mu)$. From (2), $f^{-1}(\mu)$ is $r$-fspc. Thus, $\operatorname{SPC}_{\tau_{1}}(\lambda, r)(x)$ $\leq f^{-1}(\mu)(x)=\mu(f(x))<t$. It is a contradiction to (3.9).

(3) $\Rightarrow(4)$. For all $\mu \in I^{Y}, r \in I_{\circ}$, put $\lambda=f^{-1}(\mu)$. From (3), we have

$$
f\left(\operatorname{SPC}_{\tau_{1}}\left(f^{-1}(\mu), r\right)\right) \leq \operatorname{SPC}_{T_{2}}\left(f\left(f^{-1}(\mu)\right), r\right) \leq \operatorname{SPC}_{\tau_{2}}(\mu, r) .
$$

It implies

$$
\operatorname{SPC}_{T_{1}}\left(f^{-1}(\mu), r\right) \leq f^{-1}\left(f\left(\operatorname{SPC}_{T_{1}}\left(f^{-1}(\mu), r\right)\right)\right) \leq f^{-1}\left(\operatorname{SPC}_{T_{2}}(\mu, r)\right) .
$$

$(4) \Rightarrow(5)$. It is easily proved from Theorem $2.5(5)$. 
(5) $\Rightarrow(1)$. Let $\mu$ be an $r$-fspo set of $Y$. From Theorem 2.5(2), $\mu=\mathrm{SPI}_{\tau_{2}}(\mu, r)$. By (5),

$$
f^{-1}(\mu) \leq \operatorname{SPI}_{\tau_{1}}\left(f^{-1}(\mu), r\right)
$$

On the other hand, by Theorem 2.5(4),

$$
f^{-1}(\mu) \geq \operatorname{SPI}_{\tau_{1}}\left(f^{-1}(\mu), r\right)
$$

Thus, $f^{-1}(\mu)=\operatorname{SPI}_{\tau_{1}}\left(f^{-1}(\mu), r\right)$, that is, $f^{-1}(\mu)$ is $r$-fspo.

$(1) \Rightarrow(6)$. Let $\mu \in I^{Y}$ and $r \in I_{\circ}$. According to the assumption, $f^{-1}\left(\operatorname{SPC}_{\tau_{2}}(\mu, r)\right)$ is $r$ fspc set of $X$. Hence,

$$
\begin{aligned}
f^{-1}\left(\operatorname{SPC}_{\tau_{2}}(\mu, r)\right) & \geq C_{\tau_{1}}\left(P I_{\tau_{1}}\left(f^{-1}\left(\operatorname{SPC}_{\tau_{2}}(\mu, r)\right), r\right), r\right) \\
& \geq C_{\tau_{1}}\left(P I_{\tau_{1}}\left(f^{-1}(\mu), r\right), r\right) .
\end{aligned}
$$

$(6) \Rightarrow(7)$. It can be proved by using (4), (5).

(7) $\Rightarrow(8)$. Let $\lambda \in I^{X}$ and $r \in I_{\circ}$. We put $\mu=f(\lambda)$; then $\lambda \leq f^{-1}(\mu)$. According to the assumption,

$$
\begin{aligned}
\underline{1}-I_{\tau_{1}}\left(P C_{\tau_{1}}(\underline{1}-\lambda, r), r\right) & \leq \underline{1}-I_{\tau_{1}}\left(P C_{\tau_{1}}\left(f^{-1}(\underline{1}-\mu), r\right), r\right) \\
& \leq \underline{1}-f^{-1}\left(\operatorname{SPI}_{\tau_{2}}(\underline{1}-\mu, r)\right) .
\end{aligned}
$$

Thus,

$$
C_{\tau_{1}}\left(P I_{\tau_{1}}(\lambda, r), r\right) \leq C_{\tau_{1}}\left(P I_{\tau_{1}}\left(f^{-1}(\mu), r\right), r\right) \leq f^{-1}\left(\operatorname{SPC}_{\tau_{2}}(\mu, r)\right)
$$

Hence,

$$
f\left(C_{\tau_{1}}\left(P I_{\tau_{1}}(\lambda, r), r\right)\right) \leq f f^{-1}\left(\operatorname{SPC}_{\tau_{2}}(\mu, r)\right) \leq \operatorname{SPC}_{\tau_{2}}(\mu, r)=\operatorname{SPC}_{\tau_{2}}(f(\lambda), r)
$$

(8) $\Rightarrow(1)$. Let $r \in I_{\circ}$ and let $\mu$ be an $r$-fspc set of $Y$. According to the assumption,

$$
f\left(C_{\tau_{1}}\left(P I_{\tau_{1}}\left(f^{-1}(\mu), r\right), r\right)\right) \leq \operatorname{SPC}_{\tau_{2}}\left(f f^{-1}(\mu), r\right) \leq \operatorname{SPC}_{\tau_{2}}(\mu, r)=\mu
$$

Then $C_{\tau_{1}}\left(P I_{\tau_{1}}\left(f^{-1}(\mu), r\right), r\right) \leq f^{-1} f\left(C_{\tau_{1}}\left(P I_{\tau_{1}}\left(f^{-1}(\mu), r\right), r\right)\right) \leq f^{-1}(\mu)$. Thus, $f^{-1}(\mu)$ is an $r$-fspc set of $X$, hence, $f$ is fuzzy SP irresolute continuous.

The following theorem is proved similarly as Theorem 3.5.

THEOREM 3.6. Let $(X, \tau)$ and $(Y, \eta)$ be FTS's. Let $f: X \rightarrow Y$ be a mapping. The following statements are equivalent:

(1) $f$ is fuzzy SP continuous,

(2) $f\left(\operatorname{SPC}_{\tau}(\lambda, r)\right) \leq C_{\eta}(f(\lambda), r)$, for each $\lambda \in I^{X}$ and $r \in I_{\circ}$,

(3) $\operatorname{SPC}_{\tau}\left(f^{-1}(\mu), r\right) \leq f^{-1}\left(C_{\eta}(\mu, r)\right)$, for each $\mu \in I^{Y}$ and $r \in I_{\circ}$,

(4) $f^{-1}\left(I_{\eta}(\mu, r)\right) \leq \operatorname{SPI}_{\tau}\left(f^{-1}(\mu), r\right)$, for each $\mu \in I^{Y}$ and $r \in I_{\circ}$, 
(5) $C_{T}\left(P I_{T}\left(f^{-1}(\mu), r\right), r\right) \leq f^{-1}\left(C_{\eta}(\mu, r)\right)$, for each $\mu \in I^{Y}$ and $r \in I_{\circ}$,

(6) $f\left(C_{\tau}\left(P I_{\tau}(\lambda, r), r\right)\right) \leq C_{\eta}(f(\lambda), r)$, for each $\lambda \in I^{X}$ and $r \in I_{\circ}$.

THEOREM 3.7. Let $f:(X, \tau) \rightarrow(Y, \eta)$ be a mapping from an FTS $(X, \tau)$ into an FTS $(Y, \eta)$. If $f$ is fuzzy SP irresolute continuous, then

$$
f^{-1}(\mu) \leq \operatorname{SPI}_{\tau}\left(f^{-1}\left(I_{\eta}\left(P C_{\eta}(\mu, r), r\right)\right), r\right),
$$

for each $\mu$ is an $r$-fspo in $Y$ and $r \in I_{\text {. }}$.

Proof. Let $r \in I_{\text {。 }}$ and let $\mu$ be an $r$-fspo set of $Y$. Then $f^{-1}(\mu) \leq f^{-1}\left(I_{\eta}\left(P C_{\eta}(\mu, r), r\right)\right)$. Since $f^{-1}(\mu)$ is an $r$-fspo set of $X$, we have

$$
f^{-1}(\mu) \leq \operatorname{SPI}_{\tau}\left(f^{-1}\left(I_{\eta}\left(P C_{\eta}(\mu, r), r\right)\right), r\right) .
$$

THEOREM 3.8. Let $f:(X, \tau) \rightarrow(Y, \eta)$ be a bijective mapping from an FTS $(X, \tau)$ into an FTS $(Y, \eta)$. The mapping $f$ is fuzzy SP continuous if and only if $I_{\eta}(f(\lambda), r) \leq f\left(\operatorname{SPI}_{\tau}(\lambda\right.$, $r)$ ), for each $\lambda \in I^{X}$ and $r \in I_{\text {。 }}$.

Proof. We suppose that $f$ is fuzzy SP continuous. For any $\lambda \in I^{X}$ and $r \in I_{\circ}$, $f^{-1}\left(I_{\eta}(f(\lambda), r)\right)$ is an $r$-fspo set. From Theorem 3.6(4) and the fact that $f$ is injective, we have

$$
f^{-1}\left(I_{\eta}(f(\lambda), r)\right) \leq \operatorname{SPI}_{\eta}\left(f^{-1} f(\lambda), r\right)=\operatorname{SPI}_{\tau}(\lambda, r)
$$

Again, since $f$ is surjective, we obtain

$$
I_{\eta}(f(\lambda), r)=f f^{-1}\left(I_{\eta}(f(\lambda), r)\right) \leq f\left(\operatorname{SPI}_{\tau}(\lambda, r)\right) .
$$

Conversely, let $\mu \in I^{Y}$ and $r \in I_{\circ}$ with $\eta(\mu) \geq r$. Then $I_{\eta}(\mu, r)=\mu$. According to the assumption,

$$
f\left(\operatorname{SPI}_{\tau}\left(f^{-1}(\mu), r\right)\right) \geq I_{\eta}\left(f f^{-1}(\mu), r\right)=I_{\eta}(\mu, r)=\mu .
$$

This implies that

$$
f^{-1} f\left(\operatorname{SPI}_{\tau}\left(f^{-1}(\mu), r\right)\right) \geq f^{-1}(\mu) .
$$

Since $f$ is injective, we obtain

$$
\operatorname{SPI}_{\tau}\left(f^{-1}(\mu), r\right)=f^{-1} f\left(\operatorname{SPI}_{\tau}\left(f^{-1}(\mu), r\right)\right) \geq f^{-1}(\mu) .
$$

Hence, $\operatorname{SPI}_{\tau}\left(f^{-1}(\mu), r\right)=f^{-1}(\mu)$, so $f^{-1}(\mu)$ is an $r$-fspo set. Thus, $f$ is fuzzy SP continuous.

THEOREM 3.9. Let $(X, \tau)$ and $(Y, \eta)$ be FTS's. Let $f: X \rightarrow Y$ be a mapping. The following statements are equivalent:

(1) $f$ is fuzzy SP irresolute open,

(2) $f\left(\operatorname{SPI}_{\tau}(\lambda, r)\right) \leq \operatorname{SPI}_{\eta}(f(\lambda), r)$, for each $\lambda \in I^{X}$ and $r \in I_{\circ}$, 
(3) $\operatorname{SPI}_{\tau}\left(f^{-1}(\mu), r\right) \leq f^{-1}\left(\operatorname{SPI}_{\eta}(\mu, r)\right)$, for each $\mu \in I^{Y}$ and $r \in I_{\circ}$,

(4) for any $\mu \in I^{Y}$ and any $r$-fspc $\lambda \in I^{X}$ with $f^{-1}(\mu) \leq \lambda$, there exists an $r$-fspc $\rho \in I^{Y}$ with $\mu \leq \rho$ such that $f^{-1}(\rho) \leq \lambda$.

Proof. (1) $\Rightarrow(2)$. For each $\lambda \in I^{X}$, since $\operatorname{SPI}_{\tau}(\lambda, r) \leq \lambda$ from Theorem 2.5(4), we have $f\left(\operatorname{SPI}_{\tau}(\lambda, r)\right) \leq f(\lambda)$. From $(1), f\left(\operatorname{SPI}_{\tau}(\lambda, r)\right)$ is an $r$-fspo. Hence, $f\left(\operatorname{SPI}_{\tau}(\lambda, r)\right) \leq$ $\mathrm{SPI}_{\eta}(f(\lambda), r)$.

(2) $\Rightarrow(3)$. For all $\mu \in I^{Y}, r \in I_{\circ}$, put $\lambda=f^{-1}(\mu)$ from (2). Then

$$
f\left(\operatorname{SPI}_{\tau}\left(f^{-1}(\mu), r\right)\right) \leq \operatorname{SPI}_{\eta}\left(f\left(f^{-1}(\mu)\right), r\right) \leq \operatorname{SPI}_{\eta}(\mu, r) .
$$

It implies $\operatorname{SPI}_{\tau}\left(f^{-1}(\mu), r\right) \leq f^{-1}\left(\operatorname{SPI}_{\eta}(\mu, r)\right)$.

$(3) \Rightarrow(4)$. Let $\lambda$ be an $r$-fspc set of $X$ such that $f^{-1}(\mu) \leq \lambda$. Since $\underline{1}-\lambda \leq f^{-1}(\underline{1}-\mu)$ and $\mathrm{SPI}_{\tau}(\underline{1}-\lambda, r)=\underline{1}-\lambda$,

$$
\operatorname{SPI}_{\tau}(\underline{1}-\lambda, r)=\underline{1}-\lambda \leq \operatorname{SPI}_{\tau}\left(f^{-1}(\underline{1}-\mu), r\right) .
$$

From (3),

$$
\underline{1}-\lambda \leq \operatorname{SPI}_{\tau}\left(f^{-1}(\underline{1}-\mu), r\right) \leq f^{-1}\left(\operatorname{SPI}_{\eta}(\underline{1}-\mu, r)\right) .
$$

It implies

$$
\lambda \geq \underline{1}-f^{-1}\left(\operatorname{SPI}_{\eta}(\underline{1}-\mu, r)\right)=f^{-1}\left(\underline{1}-\operatorname{SPI}_{\eta}(\underline{1}-\mu, r)\right)=f^{-1}\left(\operatorname{SPC}_{\eta}(\mu, r)\right) .
$$

Hence, there exists an $r$-fspc $\operatorname{SPC}_{\eta}(\mu, r) \in I^{Y}$ with $\mu \leq \operatorname{SPC}_{\eta}(\mu, r)$ such that $f^{-1}\left(\operatorname{SPC}_{\eta}(\mu\right.$, $r)) \leq \lambda$.

$(4) \Rightarrow(1)$. Let $\omega$ be an $r$-fspo set of $X$. Put $\mu=\underline{1}-f(\omega)$ and $\lambda=\underline{1}-\omega$ such that $\lambda$ is $r$-fspc. We obtain

$$
f^{-1}(\mu)=f^{-1}(\underline{1}-f(\omega))=\underline{1}-f^{-1}(f(\omega)) \leq \underline{1}-\omega=\lambda .
$$

From (4), there exists an $r$-fspc set $\rho$ with $\mu \leq \rho$ such that $f^{-1}(\rho) \leq \lambda=\underline{1}-\omega$. It implies $\omega \leq \underline{1}-f^{-1}(\rho)=f^{-1}(\underline{1}-\rho)$. Thus, $f(\omega) \leq f\left(f^{-1}(\underline{1}-\rho)\right) \leq \underline{1}-\rho$. On the other hand, since $\mu \leq \rho$,

$$
f(\omega)=\underline{1}-\mu \geq \underline{1}-\rho .
$$

Hence, $f(\omega)=\underline{1}-\rho$, that is, $f(\omega)$ is $r$-fspo.

The following three theorems are proved similarly as Theorem 3.9.

THEOREM 3.10. Let $(X, \tau)$ and $(Y, \eta)$ be FTS's. Let $f: X \rightarrow Y$ be a mapping. The following statements are equivalent:

(1) $f$ is fuzzy SP open,

(2) $f\left(I_{\tau}(\lambda, r)\right) \leq \operatorname{SPI}_{\eta}(f(\lambda), r)$, for each $\lambda \in I^{X}$ and $r \in I_{\circ}$, 
(3) $I_{\tau}\left(f^{-1}(\mu), r\right) \leq f^{-1}\left(\operatorname{SPI}_{\eta}(\mu, r)\right)$, for each $\mu \in I^{Y}$ and $r \in I_{\circ}$,

(4) for any $\lambda \in I^{Y}$ and any $\mu \in I^{X}$ with $\tau(\underline{1}-\mu) \geq r$ such that $f^{-1}(\lambda) \leq \mu$, there exists an $r$-fspc $\rho \in I^{Y}$ with $\lambda \leq \rho$ such that $f^{-1}(\rho) \leq \mu$.

THEOREM 3.11. Let $(X, \tau)$ and $(Y, \eta)$ be FTS's. Let $f: X \rightarrow Y$ be a mapping. The following statements are equivalent:

(1) $f$ is fuzzy SP irresolute closed,

(2) $\operatorname{SPC}_{\eta}(f(\lambda), r) \leq f\left(\operatorname{SPC}_{\tau}(\lambda, r)\right)$, for each $\lambda \in I^{X}$ and $r \in I_{\circ}$.

THEOREM 3.12. Let $(X, \tau)$ and $(Y, \eta)$ be FTS's. Let $f: X \rightarrow Y$ be a mapping. The following statements are equivalent:

(1) $f$ is fuzzy SP closed,

(2) $\operatorname{SPC}_{\eta}(f(\lambda), r) \leq f\left(C_{\tau}(\lambda, r)\right)$, for each $\lambda \in I^{X}$ and $r \in I_{\circ}$.

THEOREM 3.13. Let $f:(X, \tau) \rightarrow(Y, \eta)$ be a bijective mapping from an FTS $(X, \tau)$ into an FTS $(Y, \eta)$. Then the following statements are equivalent:

(1) $f$ is fuzzy SP irresolute closed,

(2) $f^{-1}\left(\operatorname{SPC}_{\eta}(\mu, r)\right) \leq \operatorname{SPC}_{\tau}\left(f^{-1}(\mu), r\right)$, for each $\mu \in I^{Y}$ and $r \in I_{\circ}$,

(3) $f$ is fuzzy SP irresolute open,

(4) $f^{-1}$ is fuzzy SP irresolute continuous.

Proof. $(1) \Rightarrow(2)$. Let $f$ be fuzzy SP irresolute closed. From Theorem 3.11(2), $f\left(\operatorname{SPC}_{\tau}(\lambda, r)\right) \geq \operatorname{SPC}_{\eta}(f(\lambda), r)$, for each $\lambda \in I^{X}$ and $r \in I_{\circ}$. For all $\mu \in I^{Y}, r \in I_{\circ}$, put $\lambda=f^{-1}(\mu)$ from (1). Since $f$ is onto, $f\left(f^{-1}(\mu)\right)=\mu$. Thus,

$$
f\left(\operatorname{SPC}_{\tau}\left(f^{-1}(\mu), r\right)\right) \geq \operatorname{SPC}_{\eta}\left(f\left(f^{-1}(\mu)\right), r\right)=\operatorname{SPC}_{\eta}(\mu, r) .
$$

Since $f$ is injective, it implies

$$
\operatorname{SPC}_{\tau}\left(f^{-1}(\mu), r\right)=f^{-1}\left(f\left(\operatorname{SPC}_{\tau}\left(f^{-1}(\mu), r\right)\right)\right) \geq f^{-1}\left(\operatorname{SPC}_{\eta}(\mu, r)\right) .
$$

(2) $\Rightarrow(1)$. From (2), put $\mu=f(\lambda)$ for each $\lambda \in I^{X}$. Since $f$ is injective,

$$
f^{-1}\left(\operatorname{SPC}_{\eta}(f(\lambda), r)\right) \leq \operatorname{SPC}_{\tau}\left(f^{-1}(f(\lambda)), r\right)=\operatorname{SPC}_{\tau}(\lambda, r) .
$$

Since $f$ is onto, $\operatorname{SPC}_{\eta}(f(\lambda), r) \leq f\left(\operatorname{SPC}_{\tau}(\lambda, r)\right)$. From Theorem 3.11(2), $f$ is fuzzy SP irresolute closed.

$(2) \Leftrightarrow(3)$. From Theorems 3.9(3) and 2.5(5), it is proved from the following:

$$
\begin{aligned}
& f^{-1}\left(\operatorname{SPC}_{\eta}(\mu, r)\right) \leq \operatorname{SPC}_{\tau}\left(f^{-1}(\mu), r\right) \\
\Longleftrightarrow & f^{-1}\left(\underline{1}-\operatorname{SPI}_{\eta}(\underline{1}-\mu, r)\right) \leq \underline{1}-\operatorname{SPI}_{\tau}\left(\underline{1}-f^{-1}(\mu), r\right) \\
\Longleftrightarrow & \underline{1}-f^{-1}\left(\operatorname{SPI}_{\eta}(\underline{1}-\mu, r)\right) \leq \underline{1}-\operatorname{SPI}_{\tau}\left(f^{-1}(\underline{1}-\mu), r\right) \\
\Longleftrightarrow & f^{-1}\left(\operatorname{SPI}_{\eta}(\underline{1}-\mu, r)\right) \geq \operatorname{SPI}_{\tau}\left(f^{-1}(\underline{1}-\mu), r\right) .
\end{aligned}
$$

$(2) \Leftrightarrow(4)$. From Theorem 2.5(3), it is trivial.

From the above theorems, we easily prove the following corollary. 
COROLLARY 3.14. Let $f:(X, \tau) \rightarrow(Y, \eta)$ be a bijective mapping from an FTS $(X, \tau)$ into an FTS $(Y, \eta)$. Then the following statements are equivalent:

(1) $f$ is a fuzzy SP irresolute homeomorphism,

(2) $f$ is fuzzy SP irresolute continuous and fuzzy SP irresolute open,

(3) $f$ is fuzzy SP irresolute continuous and fuzzy SP irresolute closed,

(4) $f\left(\operatorname{SPI}_{\tau}(\lambda, r)\right)=\operatorname{SPI}_{\eta}(f(\lambda), r)$, for each $\lambda \in I^{X}$ and $r \in I_{\circ}$,

(5) $f\left(\operatorname{SPC}_{\tau}(\lambda, r)\right)=\operatorname{SPC}_{\eta}(f(\lambda), r)$, for each $\lambda \in I^{X}$ and $r \in I_{\circ}$,

(6) $\operatorname{SPI}_{\tau}\left(f^{-1}(\mu), r\right)=f^{-1}\left(\operatorname{SPI}_{\eta}(\mu, r)\right)$, for each $\mu \in I^{Y}$ and $r \in I_{\circ}$,

(7) $\operatorname{SPC}_{\tau}\left(f^{-1}(\mu), r\right)=f^{-1}\left(\operatorname{SPC}_{\eta}(\mu, r)\right)$, for each $\mu \in I^{Y}$ and $r \in I_{\circ}$.

THEOREM 3.15. Let $f:(X, \tau) \rightarrow(Y, \eta)$ be a mapping from an FTS $(X, \tau)$ into an FTS $(Y, \eta)$. Then the following statements are equivalent:

(1) $f$ is fuzzy SP irresolute open,

(2) $f\left(\operatorname{SPI}_{\tau}(\lambda, r)\right) \leq I_{\eta}\left(P C_{\tau}(f(\lambda), r), r\right)$, for each $\lambda \in I^{X}$ and $r \in I_{\circ}$.

Proof. (1) $\Rightarrow(2)$. Let $\lambda \in I^{X}$ and $r \in I_{\circ}$. Then $\operatorname{SPI}_{\tau}(\lambda, r)$ is an $r$-fspo set of $X$. By (1), $f\left(\operatorname{SPI}_{\tau}(\lambda, r)\right)$ is an $r$-fspo set of $Y$. Hence,

$$
f\left(\operatorname{SPI}_{\tau}(\lambda, r)\right) \leq I_{\eta}\left(f\left(\operatorname{SPI}_{\tau}(\lambda, r)\right), r\right) \leq I_{\eta}\left(P C_{\eta}(f(\lambda), r), r\right)
$$

(2) $\Rightarrow(1)$. Let $\lambda$ be an $r$-fspo set of $X$. From $f(\lambda)=f\left(\operatorname{SPI}_{\tau}(\lambda, r)\right) \leq I_{\eta}\left(P C_{\eta}(f(\lambda), r), r\right)$ it follows that $f(\lambda)$ is an $r$-fspo set of $Y$. Hence, $f$ is fuzzy SP irresolute open.

THEOREM 3.16. Let $f:(X, \tau) \rightarrow(Y, \eta)$ be an mapping from an FTS $(X, \tau)$ into an FTS $(Y, \eta)$. Then the following statements are equivalent:

(1) $f$ is fuzzy SP irresolute closed,

(2) $C_{\eta}\left(P I_{\eta}(f(\lambda), r)\right) \leq f\left(\operatorname{SPC}_{\tau}(\lambda, r)\right)$, for each $\lambda \in I^{X}$ and $r \in I_{\circ}$.

THEOREM 3.17. Let $f:(X, \tau) \rightarrow(Y, \eta)$ be a mapping from an FTS $(X, \tau)$ into an FTS $(Y, \eta)$. Then the following statements are equivalent:

(1) $f$ is fuzzy SP open,

(2) $f\left(I_{\tau}(\lambda, r)\right) \leq I_{\eta}\left(P C_{\eta}(f(\lambda), r), r\right)$, for each $\lambda \in I^{X}$ and $r \in I_{\circ}$.

THEOREM 3.18. Let $f:(X, \tau) \rightarrow(Y, \eta)$ be a mapping from an FTS $(X, \tau)$ into an FTS $(Y, \eta)$. Then the following statements are equivalent:

(1) $f$ is fuzzy SP closed,

(2) $C_{\eta}\left(P I_{\tau}(f(\lambda), r), r\right) \leq f\left(C_{\tau}(\lambda, r)\right)$, for each $\lambda \in I^{X}$ and $r \in I_{\circ}$.

THEOREM 3.19. Let $f:(X, \tau) \rightarrow(Y, \eta)$ be a mapping. Then $f$ is fuzzy SP irresolute open if and only if for each $v \in I^{Y}$ and each $r$-fspc set $\lambda \in I^{X}, r \in I_{\circ}$, when $f^{-1}(\nu) \leq \lambda$, there exists an $r$-fspc set $\mu \in I^{Y}$ such that $v \leq \mu$ and $f^{-1}(\mu) \leq \lambda$.

Proof. Suppose that $f$ is a fuzzy SP irresolute open mapping, $v \in I^{Y}$, and $\lambda$ is an $r$-fspc set of $X, r \in I_{\circ}$, such that $f^{-1}(v) \leq \lambda$. Then, $f(\underline{1}-\lambda) \leq f\left(f^{-1}(\underline{1}-v)\right) \leq \underline{1}-v$. Since $f$ is fuzzy SP irresolute open, then $f(\underline{1}-\lambda)$ is an $r$-fspo set of $Y$. Hence,

$$
f(\underline{1}-\lambda) \leq \operatorname{SPI}_{\eta}(\underline{1}-v) .
$$


Thus

$$
\underline{1}-\lambda \leq f^{-1}(f(\underline{1}-\lambda)) \leq f^{-1}\left(\operatorname{SPI}_{\eta}(\underline{1}-v), r\right) .
$$

It follows that

$$
\lambda \geq f^{-1}\left(\underline{1}-\operatorname{SPI}_{\eta}(\underline{1}-v, r)\right)=f^{-1}\left(\operatorname{SPC}_{\eta}(\nu, r)\right) .
$$

The result follows for $\mu=\operatorname{SPC}_{\eta}(\nu, r)$.

Conversely, let $\omega$ be only $r$-fspo set of $X$. We claim that $f(\omega)$ is an $r$-fspo set of $Y$. From $\omega \leq f^{-1}(f(\omega))$ it follows that $\underline{1}-\omega \geq \underline{1}-f^{-1} f(\omega)$, where $\underline{1}-\omega$ is an $r$-fspc set of $X$. Hence, there is $v$ an $r$-fspc set of $Y$ such that $v \geq f(\underline{1}-\omega)$ and $f^{-1}(v) \leq \underline{1}-\omega$. Since $v \geq f(\underline{1}-\omega)$, it follows that $v \geq \operatorname{SPC}_{\eta}(f(\underline{1}-\omega), r)$ or $\underline{1}-v \leq \underline{1}-\operatorname{SPC}_{\eta}(f(\underline{1}-\omega), r)=$ $\operatorname{SPI}_{\eta}(f(\omega), r)$. From $f^{-1}(v) \leq \underline{1}-\omega$, we obtain $f^{-1}(\underline{1}-v) \geq \omega$ or $\underline{1}-v \geq f f^{-1}(\underline{1}-v) \geq$ $f(\omega)$. Since $f(\omega) \leq \underline{1}-v \leq \operatorname{SPI}_{\eta}(f(\omega), r)$, we have $f(\omega)=\operatorname{SPI}_{\eta}(f(\omega), r)$. Thus, $f(\omega)$ is an $r$-fspo set of $Y$, hence, $f$ is fuzzy SP irresolute open.

THEOREM 3.20. Let $f:(X, \tau) \rightarrow(Y, \eta)$ be a mapping from an FTS $(X, \tau)$ into an FTS $(Y, \eta)$. If $f$ is fuzzy SP irresolute open, then

(1) $f^{-1}\left(C_{\eta}\left(P I_{\eta}(\mu, r), r\right)\right) \leq \operatorname{SPC}_{T}\left(f^{-1}(\mu), r\right)$, for each $\mu \in I^{Y}$ and $r \in I_{\circ}$,

(2) $f^{-1}\left(C_{\eta}(\mu, r)\right) \leq \operatorname{SPC}_{\tau}\left(f^{-1}(\mu), r\right)$, for each $\mu$ an $r$-fpo set of $Y$ and $r \in I_{\circ}$.

Proof. (1) Let $\mu \in I^{Y}$ and $r \in I_{\circ}$. Then $\operatorname{SPC}_{\tau}\left(f^{-1}(\mu), r\right)$ is an $r$-fspo set of $X$. From Theorem 3.20 it follows that there exists an $r$-fspc set $\nu$ of $Y$ such that $\mu \leq \nu$ and $f^{-1}(\nu) \leq \operatorname{SPC}_{\tau}\left(f^{-1}(\mu), r\right)$. Thus

$$
f^{-1}\left(C_{\eta}\left(P I_{\eta}(\mu, r), r\right)\right) \leq f^{-1}\left(C_{\eta}\left(P I_{\eta}(\nu, r), r\right)\right) \leq f^{-1}(\nu) \leq \operatorname{SPC}_{T}\left(f^{-1}(\mu), r\right) .
$$

(2) It follows immediately from (1).

The following theorem is proved similarly as Theorem 3.20.

THEOREM 3.21. Let $f:(X, \tau) \rightarrow(Y, \eta)$ be a mapping from an FTS $(X, \tau)$ into an FTS $(Y, \eta)$. If $f$ is fuzzy SP open, then

(1) $f^{-1}\left(C_{\eta}\left(P I_{\eta}(\mu, r), r\right)\right) \leq C_{T}\left(f^{-1}(\mu), r\right)$, for each $\mu \in I^{Y}$ and $r \in I_{\circ}$,

(2) $f^{-1}\left(C_{\eta}(\mu, r)\right) \leq C_{\tau}\left(f^{-1}(\mu), r\right)$, for each $\mu$ an $r$-fpo set of $Y$ and $r \in I_{\circ}$.

ACKNOWLEDGMent. The author is very grateful to the referees for their kindness.

\section{REFERENCES}

[1] C. L. Chang, Fuzzy topological spaces, J. Math. Anal. Appl. 24 (1968), 182-190.

[2] K. C. Chattopadhyay, R. N. Hazra, and S. K. Samanta, Gradation of openness: fuzzy topology, Fuzzy Sets and Systems 49 (1992), no. 2, 237-242.

[3] K. C. Chattopadhyay and S. K. Samanta, Fuzzy topology: fuzzy closure operator, fuzzy compactness and fuzzy connectedness, Fuzzy Sets and Systems 54 (1993), no. 2, 207212.

[4] R. N. Hazra, S. K. Samanta, and K. C. Chattopadhyay, Fuzzy topology redefined, Fuzzy Sets and Systems 45 (1992), no. 1, 79-82.

[5] U. Höhle, Upper semicontinuous fuzzy sets and applications, J. Math. Anal. Appl. 78 (1980), no. 2, 659-673. 
[6] U. Höhle and A. P. Šostak, A general theory of fuzzy topological spaces, Fuzzy Sets and Systems 73 (1995), no. 1, 131-149.

[7] _ Axiomatic foundations of fixed-basis fuzzy topology, Mathematics of Fuzzy Sets, Handb. Fuzzy Sets Ser., vol. 3, Kluwer Academic Publishers, Massachusetts, 1999, pp. 123-272.

[8] Y. C. Kim, A. A. Ramadan, and S. E. Abbas, Weaker forms of continuity in Šostak's fuzzy topology, Indian J. Pure Appl. Math. 34 (2003), no. 2, 311-333.

[9] B. Krsteska, Fuzzy strongly preopen sets and fuzzy strong precontinuity, Mat. Vesnik 50 (1998), no. 3-4, 111-123.

[10] _ Some fuzzy SP-topological properties, Mat. Vesnik 51 (1999), no. 1-2, 39-51.

[11] T. Kubiak, On fuzzy topologies, Ph.D. thesis, Adam Mickiewicz University, Poznan, 1985.

[12] T. Kubiak and A. P. Šostak, Lower set-valued fuzzy topologies, Quaest. Math. 20 (1997), no. 3, 423-429.

[13] A. A. Ramadan, Smooth topological spaces, Fuzzy Sets and Systems 48 (1992), no. 3, 371375.

[14] S. E. Rodabaugh, Categorical foundations of variable-basis fuzzy topology, Mathematics of Fuzzy Sets (U. Höhle and S. E. Rodabaugh, eds.), Handb. Fuzzy Sets Ser., vol. 3, Kluwer Academic Publishers, Massachusetts, 1999, pp. 273-388.

[15] A. P. Šostak, On a fuzzy topological structure, Rend. Circ. Mat. Palermo (2) Suppl. (1985), no. $11,89-103$.

[16] _ On some modifications of fuzzy topology, Mat. Vesnik 41 (1989), no. 1, 51-64.

[17] _ Two decades of fuzzy topology: basic ideas, notion and results, Russian Math. Surveys 44 (1989), no. 6, 125-186.

[18] _ On the neighborhood structure of fuzzy topological spaces, Zb. Rad. (1990), no. 4, 7-14.

[19] M. S. Ying, A new approach for fuzzy topology. I, Fuzzy Sets and Systems 39 (1991), no. 3, 303-321.

[20] L. A. Zadeh, Fuzzy sets, Information and Control 8 (1965), 338-353.

S. E. Abbas: Department of Mathematics, Faculty of Science, South Valley University, Sohag 82524, Egypt

E-mail address: sabbas73@yahoo.com 


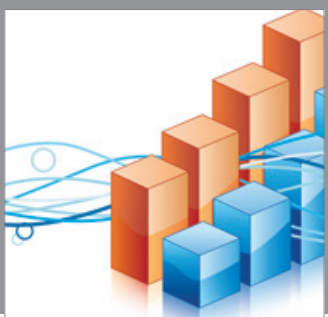

Advances in

Operations Research

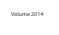

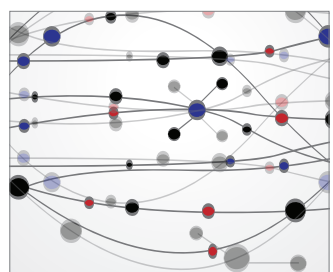

\section{The Scientific} World Journal
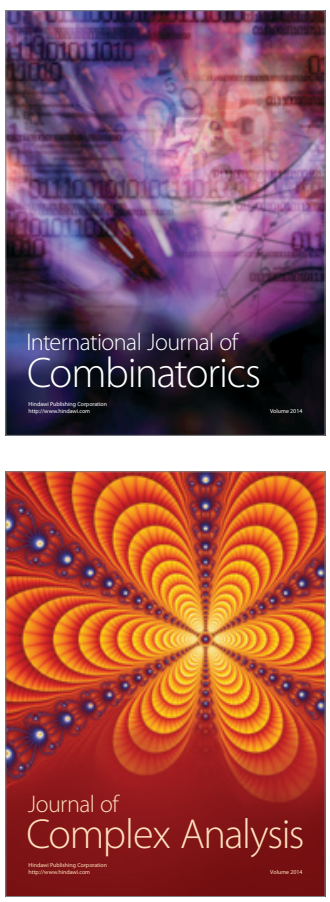

International Journal of

Mathematics and

Mathematical

Sciences
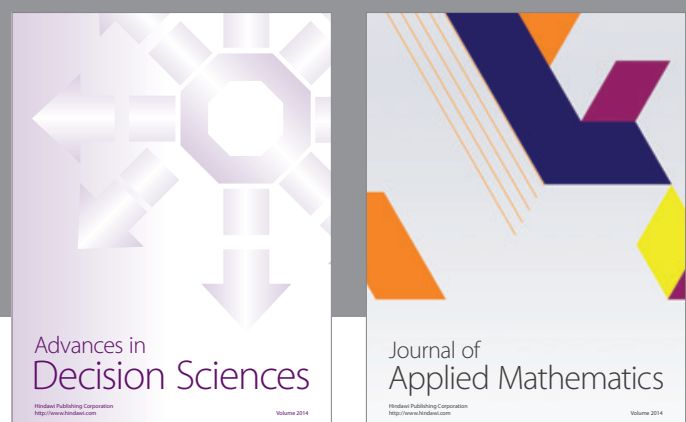

Journal of

Applied Mathematics
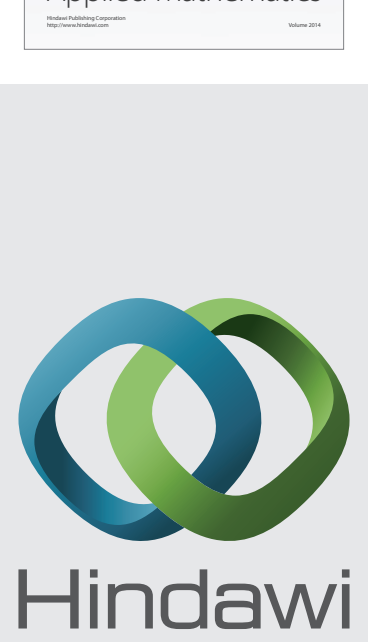

Submit your manuscripts at http://www.hindawi.com
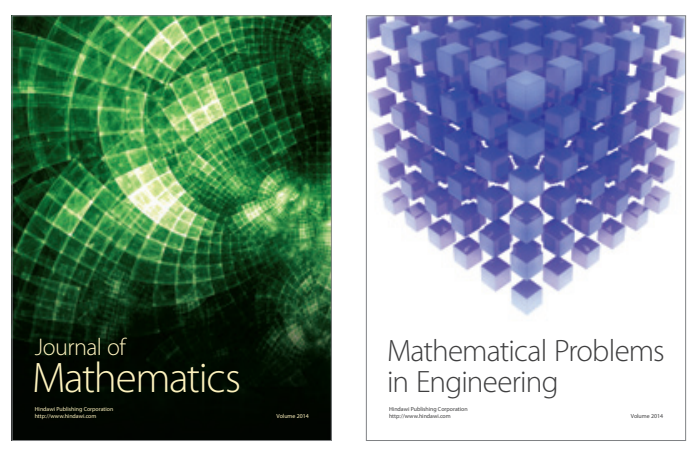

Mathematical Problems in Engineering
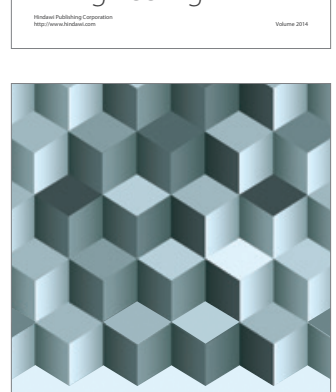

Journal of

Function Spaces
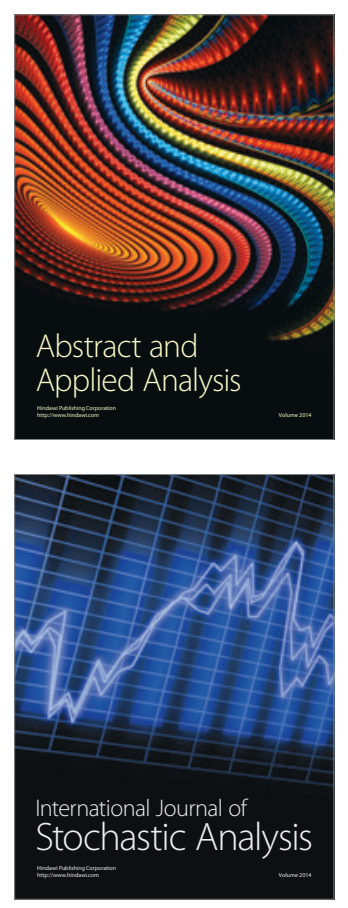

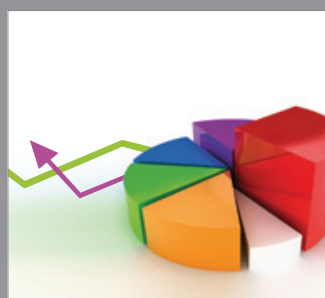

ournal of

Probability and Statistics

Promensencen
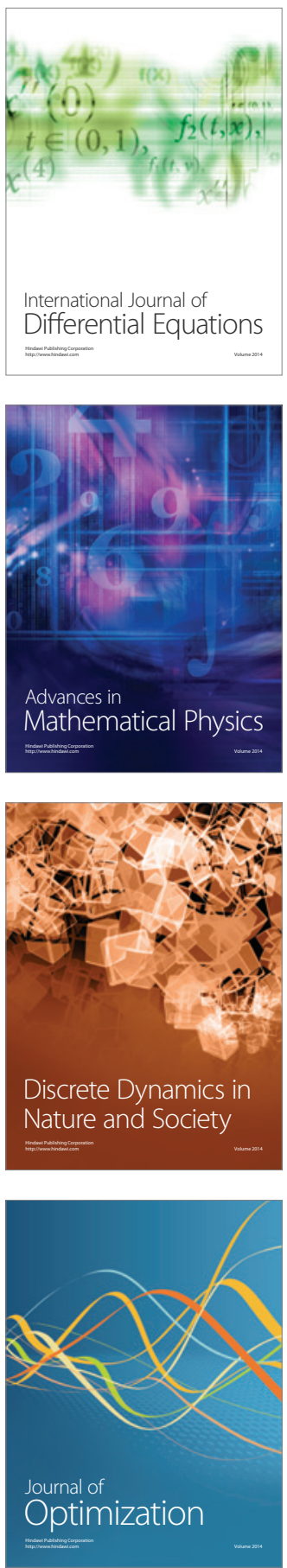